\title{
Shape Recovery of Viscoelastic Deployable Structures
}

\author{
Kawai Kwok* and Sergio Pellegrino ${ }^{\dagger}$ \\ California Institute of Technology, Pasadena, CA 91125
}

\begin{abstract}
The paper investigates the shape recovery behavior of a simple beam and a tape spring made of LDPE under prescribed deformation history at room temperature. The linear viscoelastic material properties of LDPE were measured via creep tests. An analysis of a LDPE beam under four-point bending with an imposed history of vertical deflection and reaction force was performed. A theoretical solution was constructed by employing the Alfrey's Correspondence Principle to the Euler-Bernoulli beam equation. The result was validated against a four-point bending experiment and a detailed nonlinear finite element simulation. Excellent agreement was obtained between theory, experiments and numerical simulations. A LDPE tape spring was fabricated and tested to provide an example of a simple deployable structure that recovers its deployed shape through a viscoelastic process for both equal sense and opposite sense folding.
\end{abstract}

\section{Introduction}

Deployable structures made of thin carbon fiber reinforced polymer (CFRP) composites are attractive for space missions because of their high strength to weight ratio. Current examples of this type of structures include the Boeing Springback reflectors on the MSAT and TDRS H, I, J spacecraft and the three NGST Astro Aerospace Foldable Flattenable Booms forming the Mars Advanced Radar for Subsurface and Ionosphere Sounding (MARSIS) antenna on the Mars Express spacecraft. ${ }^{1}$

Deployable space structures are often stowed for extended periods of time and subject to multiple thermal cycles during flight. Whether the temperature and deformation history will affect the shape accuracy of a structure in the deployed configuration is of great concern because its functionality is sensitive to achieving and maintaining a precise geometry. The dependence of the behavior of a composite structure on temperature and deformation history can be attributed to the viscoelasticity of the matrix material. It has been suggested that the energy stored in the MARSIS booms could not be fully recovered as a result of such temporal and thermal effects, and these booms showed more complex deployment than expected. ${ }^{2}$ Previous studies on thin CFRP deployable structures have assumed an elastic material model ${ }^{3}$ which does not capture their time and temperature dependence. Designing these structures for proper deployment requires a characterization of the viscoelastic behavior of the material and how this behavior affects their shape recovery.

Viscoelastic behavior of composites is often difficult to characterize because it involves linking the different behaviors of the fiber and matrix constituents, whose properties also vary with time and temperature. Homogenization techniques have been widely adopted to determine effective viscoelastic response of composites ${ }^{4,5}$ while micromechanical models have been suggested to study local deformations. Schapery ${ }^{6}$ found that the loss in stiffness of the matrix material over time at high temperatures allows the fibers to buckle locally and employed a micromechanical analysis to study failure in such cases. Studying the shape recovery of viscoelastic composite structures may require simulations at different scales to capture both global and local behaviors.

This research is an initial step towards achieving these goals. We consider thin beams made of homogeneous low density polyethylene (LDPE) and present analytical, numerical and experimental results of the shape recovery of a beam after being loaded for an extended period of time at room temperature. In particular, the measured reaction force, deformed shapes and curvature variations are compared to analytical and finite element predictions based on an isotropic linear viscoelastic model. We also study the viscoelastic

\footnotetext{
${ }^{*}$ Graduate Student, Graduate Aeronautical Laboratories, 1200 E. California Blvd. MC 205-45. kwk5@caltech.edu

$\dagger$ Joyce and Kent Kresa Professor of Aeronautics and Professor of Civil Engineering, Graduate Aerospace Laboratories, 1200 E. California Blvd. MC 301-46. AIAA Fellow. sergiop@caltech.edu
} 
behavior of tape springs, which are a frequently used component in deployable space structures, and present experimental observations of the shape recovery of LDPE tape springs that have been held stowed for a certain amount of time.

\section{Material Model}

We begin with a brief review of the constitutive relation for the material under study. ${ }^{7,8}$

\section{A. Linear Viscoelastic Constitutive Relation for an Isotropic Solid}

Unlike elastic solids, polymers exhibit creep or relaxation behavior under a constant applied load or deformation. For materials displaying such viscoelastic response, the modulus or compliance are represented as functions of time. Phenomenological mechanical models that consist of elastic springs and viscous dashpots are often utilized to represent the modulus and compliance functions. For example, the Maxwell model arranges a spring and a dashpot in parallel as shown in Figure 1. The modulus can be shown to be

$$
E(t)=E e^{-(t / \rho)}
$$

where $\rho$ is the relaxation time and is related to the viscosity through the relation, $\rho=\mu / E$.

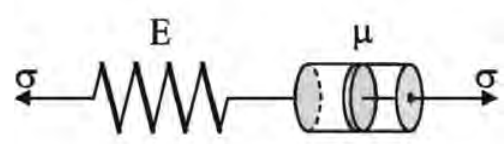

Figure 1: Maxwell model.

Real viscoelastic solids operating over a wide range of time scales can be characterized with multiple relaxation times and hence it is necessary to use several spring and dashpots to represent their behavior. The Wiechert model shown in Figure 2 is a generalized model that includes an arbitrary number of springs and dashpots. The modulus in this case is written as

$$
\left.E(t)=E_{\infty}+\sum_{i=1}^{n} E_{i} e^{-\left(t / \rho_{i}\right)}\right)
$$

where $E_{\infty}$ is the long term modulus, $n$ is the number of terms and $\rho_{i}$ is the $i$ th relaxation time. Similarly, the compliance can be formulated as

$$
D(t)=D_{0}+\sum_{j=1}^{n} D_{j}\left(1-e^{-\left(t / \tau_{j}\right)}\right)
$$

where $D_{0}$ is the instantaneous compliance and $\tau_{j}$ is the $j$ th retardation time. This type of representation is also known as a Prony series and will be employed in this study.

Viscoelastic behavior has also been shown to depend on temperature, which affects the material response by changing the time scale over which it creeps or relaxes. The correspondence between time and temperature is captured by the Time-Temperature Superposition Principle. ${ }^{7}$ This is represented mathematically by replacing the real time $t$ in Equations (2) and (3) by the reduced time $\psi$ that takes the form

$$
\psi(t)=\int_{0}^{t} \frac{d \xi}{a_{T}(T)}
$$

where $a_{T}$ is the temperature shift factor that needs to be determined experimentally.

Because all events over the history of a viscoelastic material contribute to the current state of stress and strain, the constitutive relations need to be written as an integral in time. Using Boltzmann's Superposition Principle, ${ }^{8}$ it can be shown that the uniaxial constitutive equation has the form

$$
\sigma(t)=\int_{0}^{t} E(\psi(t)-\psi(\tau)) \frac{d \epsilon(\tau)}{d \tau} d \tau
$$




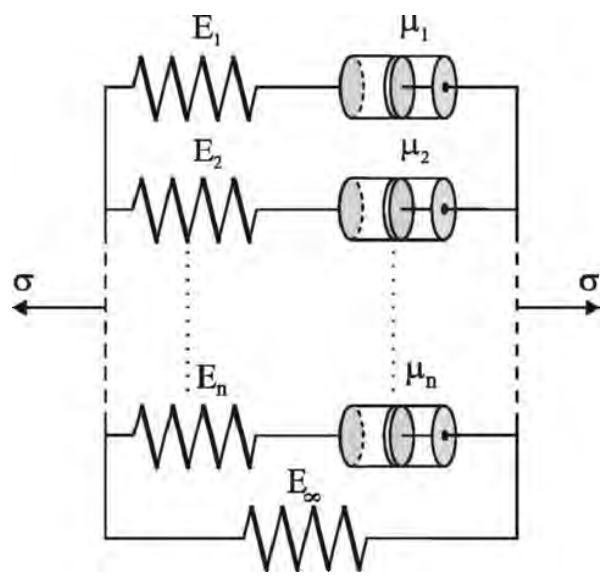

Figure 2: Wiechert Model.

$$
\epsilon(t)=\int_{0}^{t} D(\psi(t)-\psi(\tau)) \frac{d \sigma(\tau)}{d \tau} d \tau
$$

For an isotropic solid, the uniaxial constitutive relation can be extended to a three-dimensional model cast in terms of deviatoric and dilatational deformation. The corresponding constitutive relations become

$$
\begin{gathered}
s_{i j}(t)=2 \int_{0}^{t} G(\psi(t)-\psi(\tau)) \frac{\partial e_{i j}(\tau)}{\partial \tau} d \tau \\
\sigma_{k k}(t)=3 \int_{0}^{t} K(\psi(t)-\psi(\tau)) \frac{\partial \epsilon_{k k}(\tau)}{\partial \tau} d \tau
\end{gathered}
$$

where $s_{i j}$ and $e_{i j}$ are the deviatoric stress and strain components, $\sigma_{k k}$ and $\epsilon_{k k}$ are the dilatational stress and strain, $G$ is the shear modulus and $K$ is the bulk modulus. These two material functions are related to the uniaxial modulus through the equations

$$
\begin{aligned}
G(t) & =\frac{E(t)}{2(1+\nu)} \\
K(t) & =\frac{E(t)}{3(1-2 \nu)}
\end{aligned}
$$

where $\nu$ is the Poisson's ratio. The Poisson's ratio has been assumed to be constant in time to allow for a more convenient representation of shear and bulk moduli. It was found empirically that the Poisson's ratio for the material under study has a much weaker dependence on time than the uniaxial modulus, hence such an assumption is reasonably justified.

\section{B. Characterization of Low Density Polyethylene}

The material under study was characterized through a series of creep tests performed with an Instron System 5569 material testing machine, to determine $D_{0}, D_{j}$, and $\tau_{j}$ at a room temperature of $22^{\circ} C$. Test coupons with a length of $165 \mathrm{~mm}$ and a width of $40.0 \mathrm{~mm}$ were cut with a shearing machine from a LDPE sheet with a thickness of $1.56 \mathrm{~mm}$. The specimen was clamped inside an environmental chamber (Instron Heatwave Model 3119-506) utilizing a built-in thermocouple to control the temperature with a resolution of $\pm 1^{\circ} \mathrm{C}$. In addition, a type- $T$ thermocouple (made of Copper/Constantan and supplied by Omega Engineering, Inc.) was attached to the surface of a dummy LDPE specimen close to the test specimen to monitor the actual specimen temperature. As a test for stable temperature conditioning inside the environmental chamber, a temperature impulse was imposed and the subsequent temperature variation over time measured by the builtin thermocouple and the dummy specimen thermocouple were recorded. It was found that the temperature readings from the two thermocouples became identical 30 minutes after the impulse. This indicates that 
thermal equilibrium could be established within such time frame, and this thermal conditioning time was allowed prior to each test.

Stress could appear even before any imposed loading, because of thermal expansion or contraction of the clamped specimens. Therefore the position of the testing machine crosshead was adjusted so that a zero load was obtained again. To further reduce possible experimental errors, another 30 minutes were allowed to ensure the transient effects due to crosshead adjustment had disappeared. At this point, the specimens were loaded in tension at a rate of $0.1 \mathrm{MPa} / \mathrm{s}$ until a stress of $0.5 \mathrm{MPa}$ had been reached and this stress value was held constant for 3 hours. The longitudinal and transverse strains in the specimen were measured using two laser extensometers (Electronic Instrument Research Ltd LE-05) with a recording rate of $5 \mathrm{~Hz}$.

Experimental data were fitted with a three term Prony series using a nonlinear optimization algorithm. Figure 3 shows the compliance of LDPE over 4 decades of logarithmic time and Table 1 summarizes the corresponding material parameters.

\begin{tabular}{c|c|c}
\hline \hline$j$ & $D_{j}\left[P a^{-1}\right]$ & $\tau_{j}[\mathrm{~s}]$ \\
\hline 0 & $2.97 E-9$ & - \\
1 & $1.89 E-9$ & 29.35 \\
2 & $1.45 E-9$ & 413.41 \\
3 & $1.08 E-9$ & 5100.68 \\
\hline \hline
\end{tabular}

Table 1: LDPE Prony series parameters.

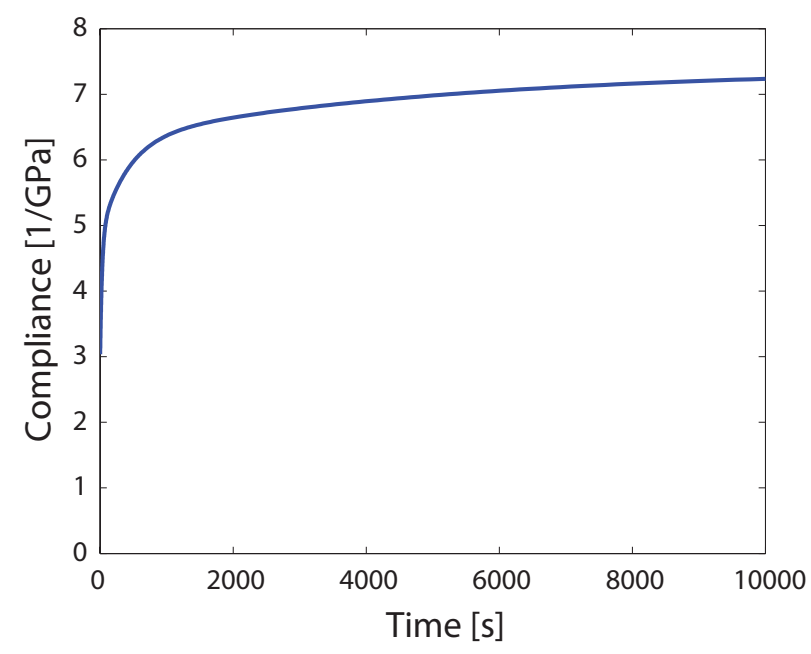

Figure 3: Creep compliance for LDPE at $22^{\circ} \mathrm{C}$.

Since the compliance of LDPE was obtained from creep tests, a conversion to modulus is needed. This was done by numerically solving the following relation,

$$
\int_{0}^{t} E(t-\tau) \frac{d D(\tau)}{d \tau} d \tau=1
$$

The necessary material functions $G$ and $K$ can now be determined from $E$ using Equations (9) and (10). It should be noted that the present material model characterizes the behavior of LDPE over 4 decades of logarithmic time at a temperature of $22^{\circ} \mathrm{C}$. For studying the shape recovery of deployable structures, the material viscoelastic response for wider time and temperature ranges is needed. Viscoelastic behavior beyond this time range can be characterized by following the creep test protocol described above, but at several different temperatures and converting all of the results to a single temperature, using the TimeTemperature Superposition Principle. 


\section{Bending of a Viscoelastic Beam}

Having determined the linear viscoelastic material model for LDPE, we turn to the shape recovery behavior of a thin LDPE beam under four-point bending and subjected to a prescribed deformation and loading history. A viscoelastic beam is one of the simplest examples of a deployable structure, whose response can be obtained analytically. It is thus a suitable problem for verifying the viscoelastic material model against experiments and finite element simulations.

Consider half of a symmetric beam as shown in Figure 4, with a simple support at point $O$ and a vertical line through point B defining the symmetry plane. We divide the analysis into four stages, namely loading, holding, unloading and recovery, and prescribe the time history of vertical deflection $d(t)$ and reaction force $R(t)$ at point $\mathrm{A}$ as follows,

$$
\begin{aligned}
\text { Loading } & : d(t)=\dot{d} t, \quad 0 \leq t \leq t_{l} \\
\text { Holding } & : d(t)=d_{0}, \quad t_{l} \leq t \leq t_{h} \\
\text { Unloading } & : d(t)=d_{0}-\dot{d}\left(t-t_{h}\right), \quad t_{h} \leq t<t_{u} \\
\text { Recovery } & : \quad R(t)=0, \quad t_{u} \leq t \leq t_{r}
\end{aligned}
$$

where the time $t$ is measured in seconds and the deflection $d(t)$ is measured in millimeters. The time history is shown schematically in Figure 5.

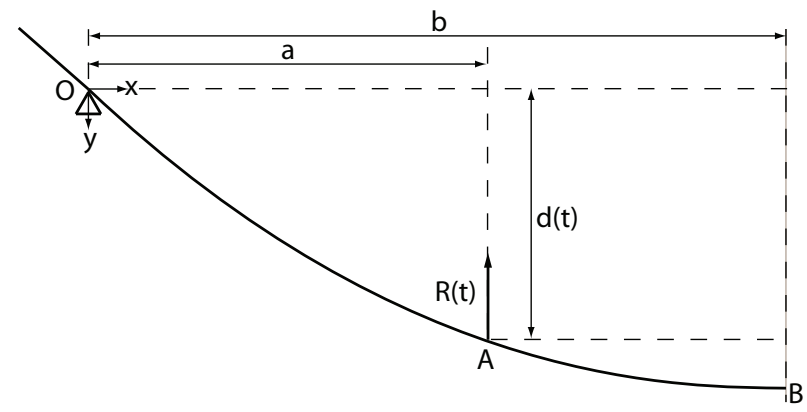

Figure 4: Schematic of half of a symmetric four-point bending test.

Such prescribed loading and boundary conditions imitate those on deployable structures in real missions, where the structures are first packaged by folding, then stored in a launch vehicle, and finally allowed to deploy freely in space. We are thus interested in finding the reaction force $R(t)$ at point A during the holding stage, the beam deflection $y(x, t)$ and the mid point curvature $\kappa(t)$ in the recovery stage.

\section{A. Theoretical Analysis}

The deflection over time of a viscoelastic beam under bending can be obtained in closed form linearized kinematics are assumed. The well known Euler-Bernoulli beam equation is given by

$$
\frac{d^{2} y(x, t)}{d x^{2}}=\frac{M(x, t)}{E(t) I}
$$

where $y(x, t)$ is the vertical deflection, $E(t)$ is the uniaxial modulus and $I$ is the second moment of area of the beam cross section.

Using Alfrey's Correspondence Principle ${ }^{9}$ the viscoelastic equivalent to the deflection equation given by Equation (12) can be developed in the Laplace domain by replacing the appropriate variables by their Laplace transform,

$$
\frac{d^{2} y(x, s)}{d x^{2}}=\frac{R(s) x}{I} s D(s), \quad 0 \leq x \leq a
$$




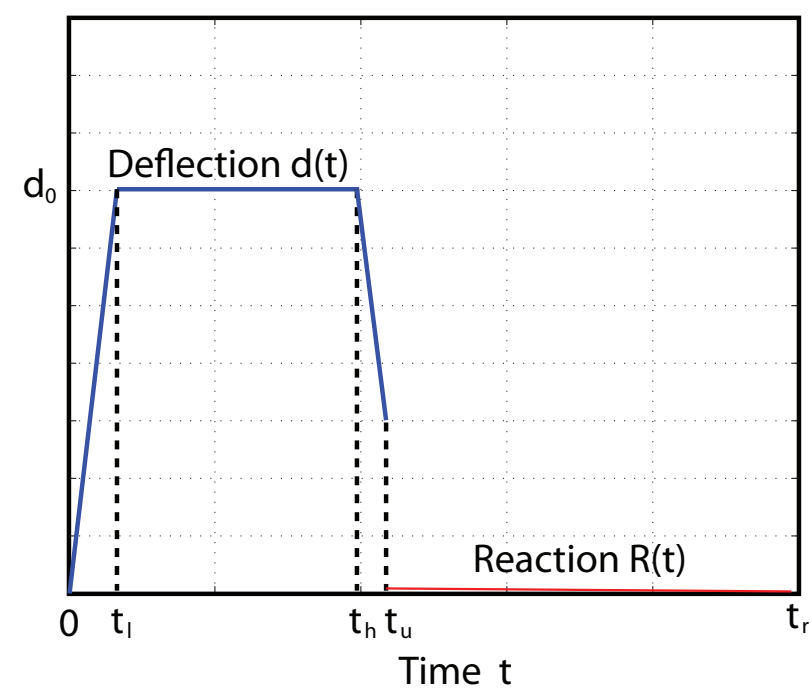

Figure 5: Imposed deflection and reaction force histories.

$$
\frac{d^{2} y(x, s)}{d x^{2}}=\frac{R(s) a}{I} s D(s), \quad a \leq x \leq b
$$

where $s$ is the Laplace variable, $D(s)$ is the uniaxial compliance and $R(s)$ is the reaction force at point A in the Laplace domain. By solving Equations (13) and (14) with the appropriate boundary conditions, we find the reaction force to be

$$
R(s)=\frac{3 I}{2 a^{3}-3 a^{2} b} s E(s) d(s)
$$

The beam deflection in the region of uniform moment is found to be

$$
y(x, s)=\frac{3 a x^{2}-6 a b x+b^{3}}{6 I} s D(s) p(s), \quad a \leq x \leq b
$$

Taking the inverse Laplace transform, we obtain

$$
\begin{gathered}
R(t)=\frac{3 I}{2 a^{3}-3 a^{2} b} \int_{0}^{t} E(t-\tau) \frac{d d(\tau)}{d \tau} d \tau \\
y(x, t)=\frac{3 a x^{2}-6 a b x+b^{3}}{6 I} \int_{0}^{t} D(t-\tau) \frac{d R(\tau)}{d \tau} d \tau, \quad a \leq x \leq b
\end{gathered}
$$

It should be noted at this point that Equations (17) and (18) involve finding time integrals from $t=0$ to the time of interest. However, as illustrated in Figure 5, neither the deflection $d(t)$ nor the reaction force $R(t)$ were specified for the entire history. In fact, a combination of both traction and displacement boundary conditions was present. Therefore in the following we will split the analysis into two time intervals.

We consider first the solution to Equation (17) from loading to the end of the unloading period. The loading and unloading times are often much smaller than the holding and recovery times. Hence the deflection $d(t)$ at point A can be closely represented by an impulse function as shown in Figure 6, where the ramp loading and unloading in Figure 5 are approximated by instantaneous jumps, and is written as

$$
d(t)=d_{0} H(t)-d_{0} H\left(t-t_{h}\right), \quad 0 \leq t \leq t_{h}
$$

where $H(t)$ is the Heaviside step function. The error resulting from such an approximation was assessed by numerical simulations and the difference in the calculated reaction force was negligible. Solving Equation (17) with the imposed deflection given by Equation (19), the reaction force during the holding period is 


$$
R(t)=\frac{3 I}{2 a^{3}-3 a^{2} b} E(t) d_{0}, \quad 0 \leq t \leq t_{h}
$$

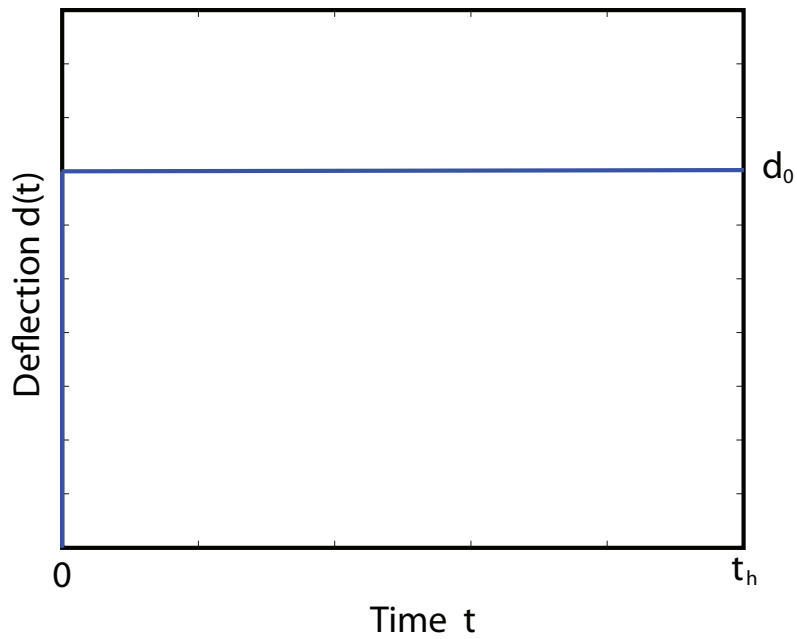

Figure 6: Deflection approximated as an impulse function.

The reaction force determined for the holding period can now be combined with the zero reaction force condition for the recovery stage to characterize the complete history, as shown in Figure 7. It is mathematically expressed as

$$
R(t)=\frac{3 I}{2 a^{3}-3 a^{2} b} E(t) d_{0}\left(1-H\left(t-t_{h}\right)\right), \quad 0 \leq t \leq t_{r}
$$

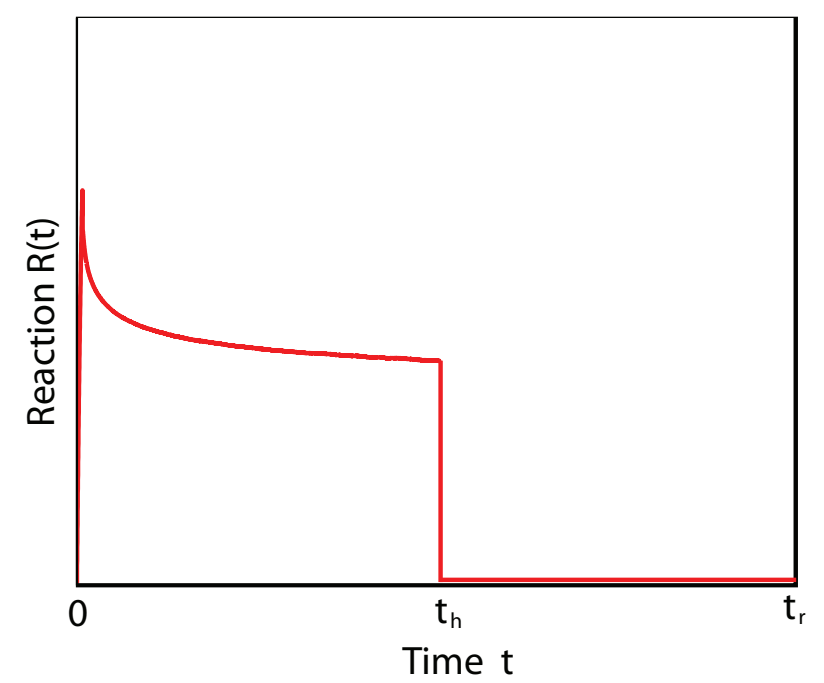

Figure 7: Complete history of reaction force.

By solving Equation (18) with the reaction force input given by Equation (21), the beam deflection and curvature in the region of uniform moment during the recovery stage are determined to be

$$
y(x, t)=\frac{3 a x^{2}-6 a b x+b^{3}}{4 a^{3}-6 a^{2} b} d_{0}\left(1-D\left(t-t_{h}\right) E\left(t_{h}\right)-\int_{t_{h}}^{t} D(t-\tau) \frac{d E(\tau)}{d \tau} d \tau\right)
$$




$$
\kappa(t)=\frac{3}{2 a^{2}-3 a b} d_{0}\left(1-D\left(t-t_{h}\right) E\left(t_{h}\right)-\int_{t_{h}}^{t} D(t-\tau) \frac{d E(\tau)}{d \tau} d \tau\right)
$$

The deflection $d(t)$ at point $\mathrm{A}$ at any instant in time is shown in Figure 8.

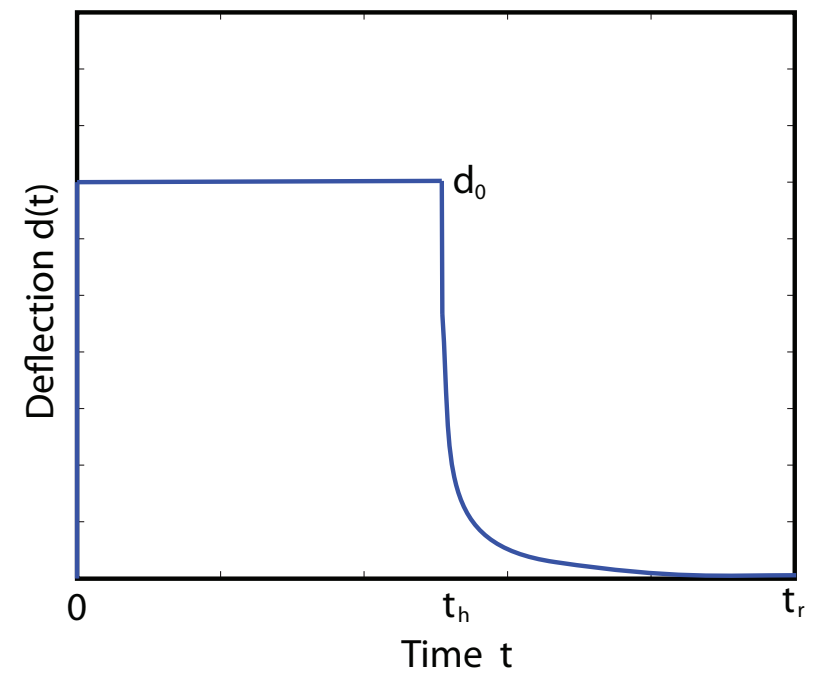

Figure 8: Time response of deflection.

\section{B. Experimental Study}

To verify the theoretical prediction, experiments were carried out on a viscoelastic beam with the aid of a four-point bending fixture. The test setup is shown in Figure 9. The specimen used was a rectangular LDPE strip with a length of $170 \mathrm{~mm}$ and a width of $13.0 \mathrm{~mm}$. The parameters that specify the deformation and reaction histories used in the experiment are summarized in Table 2.

\begin{tabular}{c|c}
\hline \hline$t_{l}$ & $8.0[\mathrm{~s}]$ \\
\hline$t_{h}$ & $1008.0[\mathrm{~s}]$ \\
\hline$t_{u}$ & $1012.5[\mathrm{~s}]$ \\
\hline$t_{r}$ & $10000[\mathrm{~s}]$ \\
\hline$\dot{d}$ & $1.0[\mathrm{~mm} / \mathrm{s}]$ \\
\hline$d_{0}$ & $8.0[\mathrm{~mm}]$ \\
\hline \hline
\end{tabular}

Table 2: Deformation and reaction force history definitions.

The reaction force and images of the specimen were recorded respectively with a load cell and a high definition camcorder over the course of experiment. Prior to the experiment, the orientation of the camcorder was adjusted until there was no distortion in the image of a square taken by the camcorder. This ensured that the camcorder was parallel to the plane of motion of the specimen. A resolution of $1920 \times 1080$ pixels and a frame rate of 30 frames per second were used. Figures 10 and 11 display the beam deformed profiles recorded during the experiment.

The coordinates of the specimen centerline were determined from the images by first tracing the top and bottom edges of the beam using the Canny edge detection algorithm in MATLAB. The centerline is then obtained by averaging the two traced edges. The resulting pixel coordinates were subject to quantization error, therefore a sinc interpolation was used to reconstruct the continuous centerline coordinates. The curvature distribution of the beam was then determined by finding the radius of the osculating circle along the centerline. 


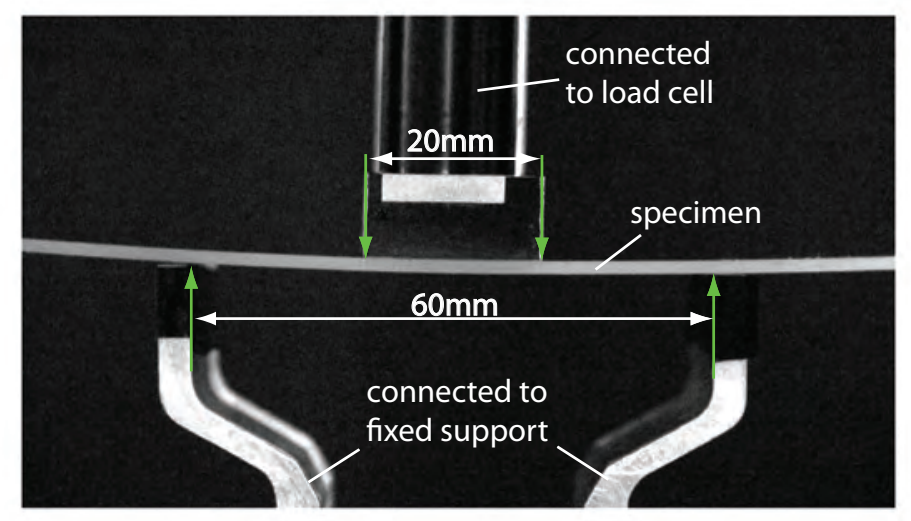

Figure 9: Beam at initial configuration.

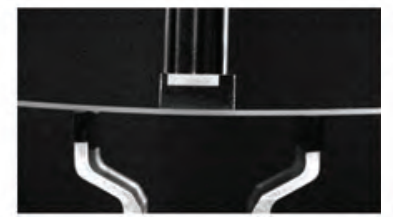

$\mathrm{t}=0 \mathrm{~s}$

1

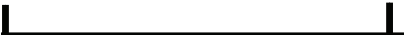

Loading

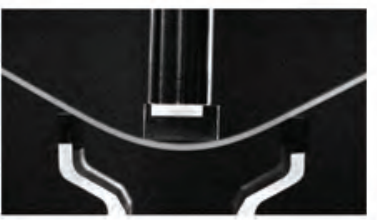

$\mathrm{t}=8 \mathrm{~s}$

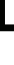

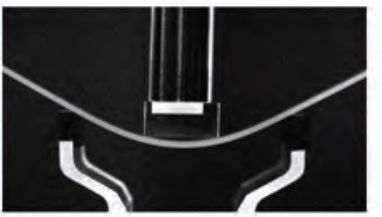

$\mathrm{t}=1008 \mathrm{~s}$

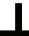

Holding

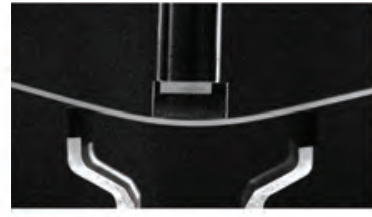

$\mathrm{t}=1012.5 \mathrm{~s}$

Figure 10: Deformed shapes from loading to unloading stages.

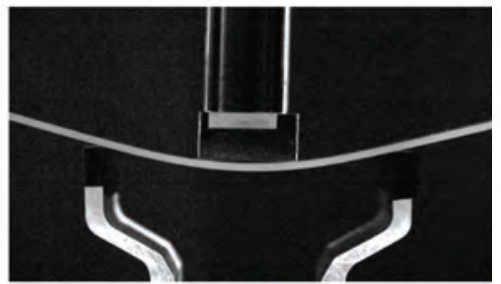

(a)

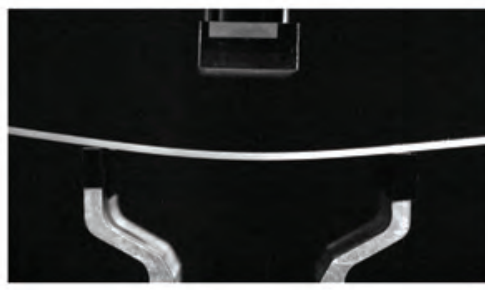

(d)

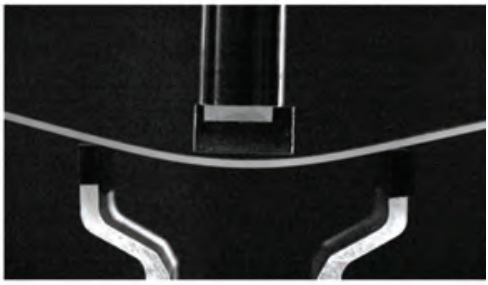

(b)

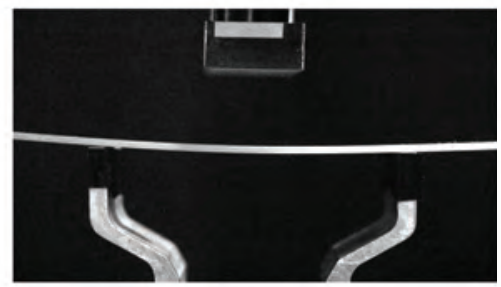

(e)

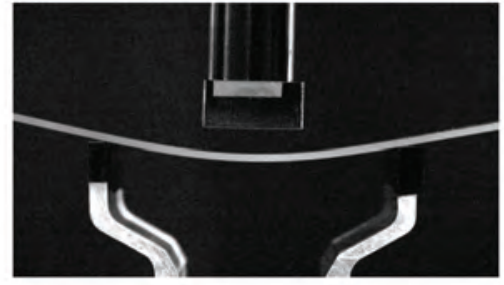

(c)

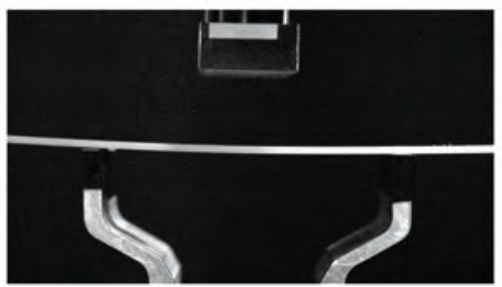

(f)

Figure 11: Deformed shapes during recovery: (a) during holding; (b) $1 \mathrm{~s}$ after unfolding; (c) $10 \mathrm{~s}$ after unfolding; (d) $100 \mathrm{~s}$ after unfolding; (e) $1000 \mathrm{~s}$ after unfolding; (f) $10000 \mathrm{~s}$ after unfolding. 


\section{Finite Element Simulation}

To assess the effect of ramp loading and unloading as well as contact friction between the fixture and the beam, a finite element simulation of a LDPE strip subject to the deformation history described above was carried out using the commercial package ABAQUS/Standard. ${ }^{10}$ Figure 12 shows the finite element model used in the simulation. The viscoelastic properties of LDPE obtained in Section 2 were assigned to the elements with the option *VISCOELASTIC, TIME=PRONY. The strip was modeled with 688 full integration 4-node shell elements (ABAQUS element $S 4$ ). The contact points between the beam and the four-point bending fixture were modeled as rigid cylindrical surfaces with a radius that is small compared to the length of the beam, and a contact interaction was enforced between the fixture and the strip. The coefficient of friction used was 0.15 , which is a nominal value for contact between plastics and aluminum.

The bottom loading fixture remains stationary throughout the simulation. To impose the deflection history on the strip, displacement boundary conditions were applied to a node that is tied to the two cylindrical contact surfaces that form the top loading fixture. A geometrically nonlinear quasi-static analysis (*VISCO option) that lasts for $10000 \mathrm{~s}$ was then carried out to find the deformed shapes and reaction force on the strip. The curvature distribution of the beam was calculated from the deformed nodal coordinates using the method described in the previous section.

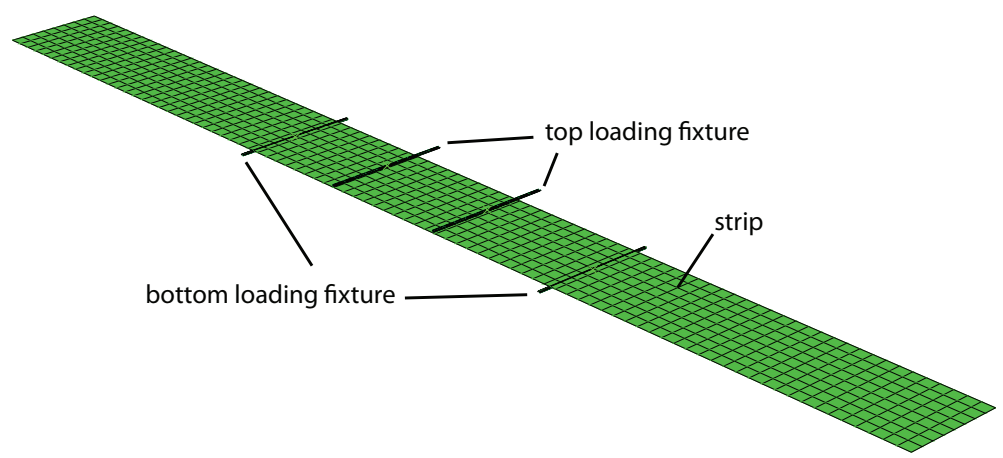

Figure 12: Finite element model for four-point bending simulation.

\section{Results and Discussion}

Comparisons between analytical predictions, finite element simulation and experimental results are shown in Figures 13 to 15. As shown in Figure 13(a), there is generally good agreement between all sets of results. During the holding period, the reaction force shows the expected relaxation behavior because a constant deflection is imposed as a condition. The analytical reaction force response differs from the experimental and finite element results during the first 8 seconds because the loading time was assumed to be instantaneous in the theoretical analysis. This transient effect produces no apparent difference in the reaction force beyond the loading period as shown in Figure 13(b).

The beam deflections in the region of uniform moment at different times during the recovery stage are shown in Figure 14. We have also compared the curvature variation of the beam, Figure 15, to ensure that all the geometric details are correctly modeled. Since the curvature distribution along the strip was found to be uniform along the strip, it is sufficient to consider only the curvature variation over time. It can be seen that excellent agreement is again obtained between theoretical, experimental and finite element results for the deformed shapes and curvature response.

\section{Shape Recovery of a Viscoelastic Tape Spring}

Having established a correct material model and finite element implementation for a viscoelastic beam under bending, we turn to the study of a viscoelastic tape spring. A tape spring is a thin cylindrical shell structure such as a steel tape measure. Tape springs have been used for many years as components of deployable spacecraft structures ${ }^{1}$ and therefore can be used as a prototypical example for studying the viscoelastic behavior in deployable structures. Packaging of a tape spring can be either by folding it in an 


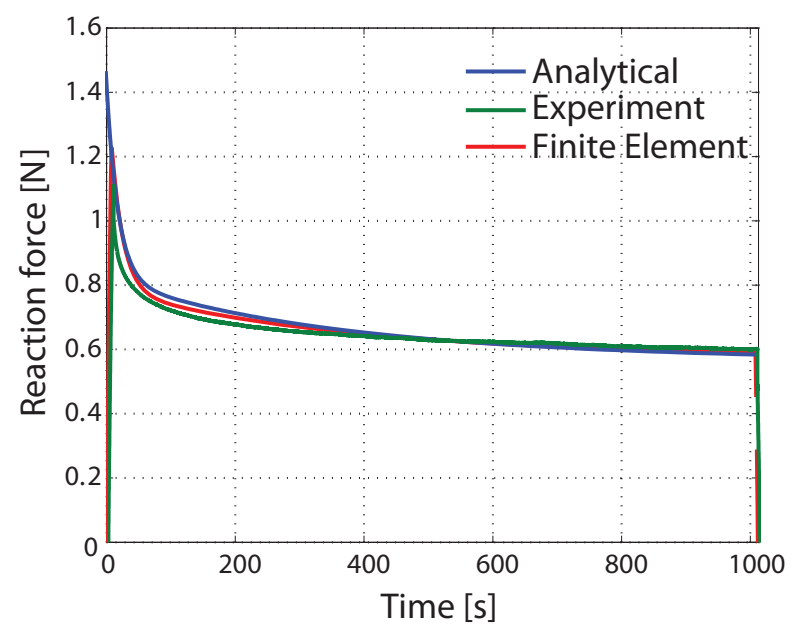

(a)

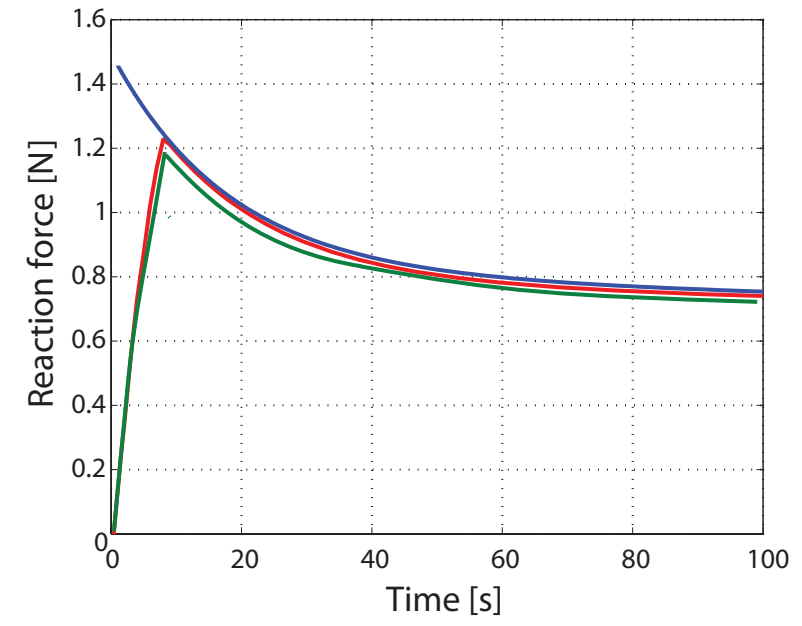

(b)

Figure 13: Reaction force response over time: (a) during holding; (b) detailed view of loading period.

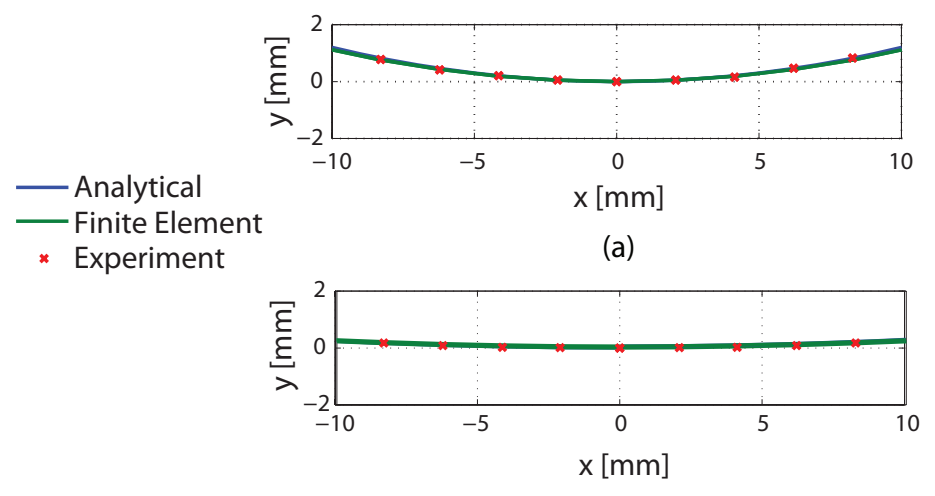

(c)

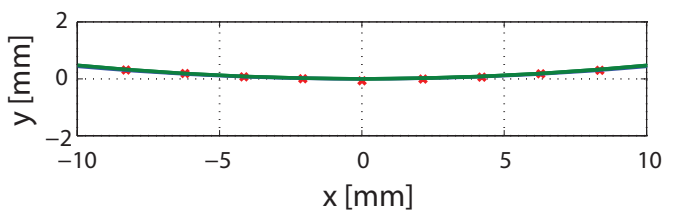

(b)

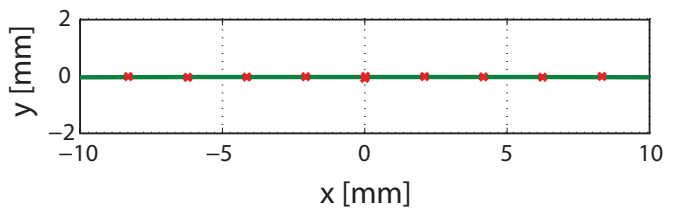

(d)

Figure 14: Deformed profiles during recovery: (a) during holding; (b) $10 \mathrm{~s}$ after unloading; (c) $100 \mathrm{~s}$ after unloading; (d) $10000 \mathrm{~s}$ after unloading. 


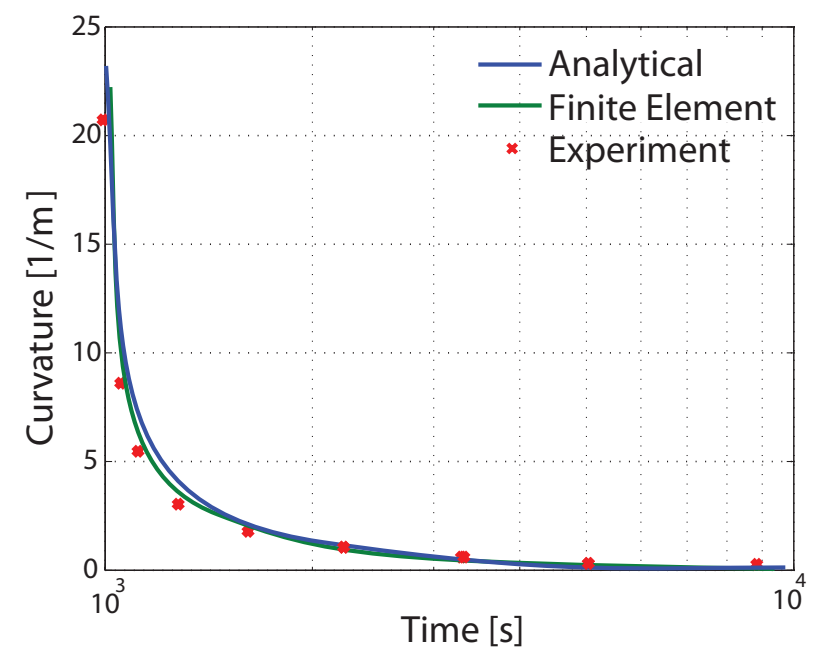

Figure 15: Curvature variation as a function of recovery time.

equal sense, i.e. inducing compressive strains along the edges as shown in Figure 16(a), or in an opposite sense as shown in Figure 16(b).

Here we consider a viscoelastic tape spring made of LDPE that is packaged into a rolled shape and held in this configuration for an extended period of time. We observe its change in shape over time after releasing the restraint that holds it rolled.

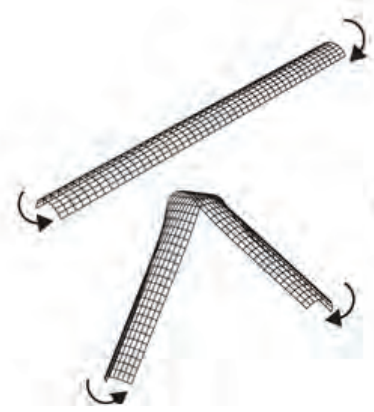

(a)

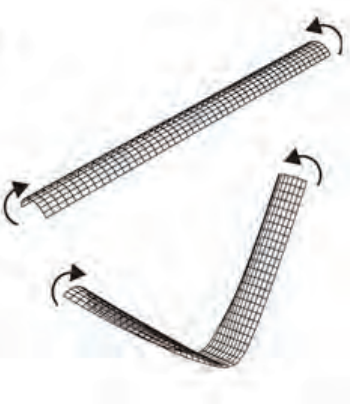

(b)

Figure 16: Two ways of bending a tape spring: (a) equal sense and (b) opposite sense.

\section{A. Fabrication Method}

The tape springs used in the present study were fabricated from flat LDPE sheets. Forming a curved LDPE sheet requires subjecting the flat sheet to a thermal remolding process determined by the characteristic temperature of the material.

Prior to the thermal remolding process, a $340 \mathrm{~mm}$ long, $75 \mathrm{~mm}$ wide and $0.7 \mathrm{~mm}$ thick rectangular sheet was cut from a sheet of LDPE. The mold used consisted of a stainless steel tube with a diameter of $38 \mathrm{~mm}$ and a $0.4 \mathrm{~mm}$ thick, curved steel sheet with a circular cross section with the same diameter as the tube. Both parts of this mold were smoothly polished with diamond grit. The LDPE sheet was sandwiched between sheets of PTFE release fabric and wrapped around the steel tube. The curved steel sheet was put on top of this sandwich, see Figure 17. The whole assembly was tightly restrained with heat shrink tape and the final package is shown in Figure 17.

The processing temperature for the remolding of LDPE, which is an uncrosslinked polymer, is its melting 
temperature. Since this temperature was not known, several attempts were performed and a temperature of $120^{\circ} \mathrm{C}$ was found to be adequate. The package was heated to $120^{\circ} \mathrm{C}$ and maintained at this temperature for 4 hours inside an oven with a temperature control precision of $\pm 2^{\circ} \mathrm{C}$. This process allowed enough time for the LDPE to recrystallize. Then the assembly was allowed to cool to room temperature over 8 hours. A relatively long cooling time was used to minimize the effect of physical ageing and the change in material properties due to the thermal process.

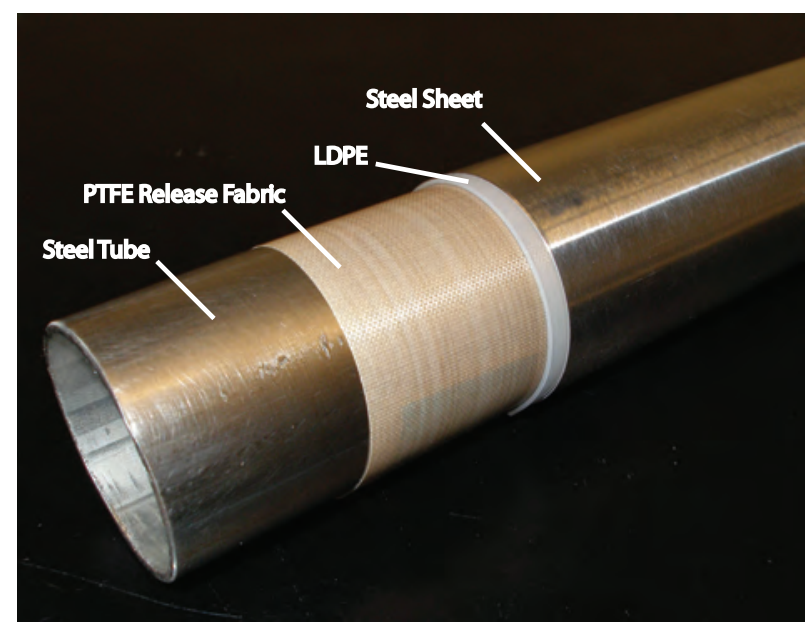

Figure 17: Assembly of LDPE sheet and mold.

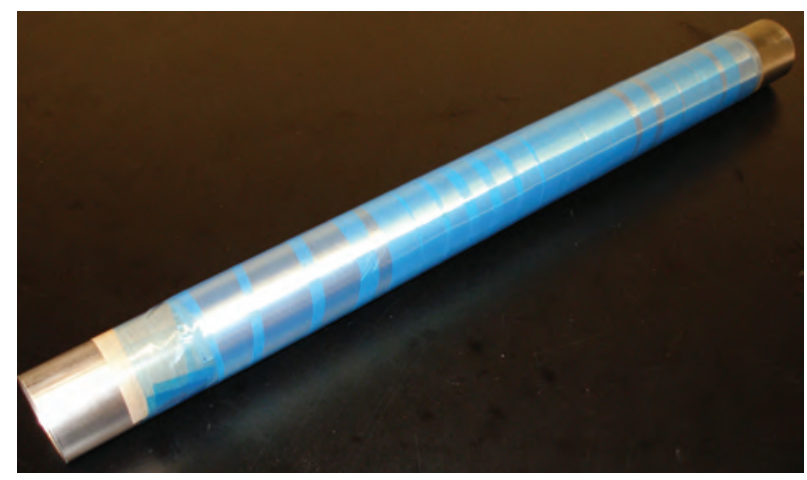

Figure 18: Assembly restrained by heat shrink tape.

The fabricated tape spring is shown in Figure 19. A survey of thickness measurements at different locations on the tape spring demonstrated a good thickness uniformity.

\section{B. Experimental Study}

Experiments were performed to observe the shape recovery of a folded tape spring. The LDPE tape spring was attached at one end to a fixed stand, with the convex side facing down as shown in Figure 19. Folding was achieved by slowly rolling a solid cylinder, with a diameter of $38 \mathrm{~mm}$, over the tape spring along the meridional direction. This process took $60 \mathrm{~s}$. The solid cylinder was then removed and the tape spring was held in the resulting folded configuration using steel clips. After $1000 \mathrm{~s}$ the clips were quickly released and this allowed the tape spring to start deploying. The change in shape of the tape spring over time was recorded using a high resolution camcorder. Figures 21 and 22 illustrate the recovery behavior for equal and opposite sense folding.

For the case of opposite sense folding the tape spring initially maintained a zero mean curvature. The region of zero mean curvature then localized and finally disappeared, at which point the tape spring recovered 


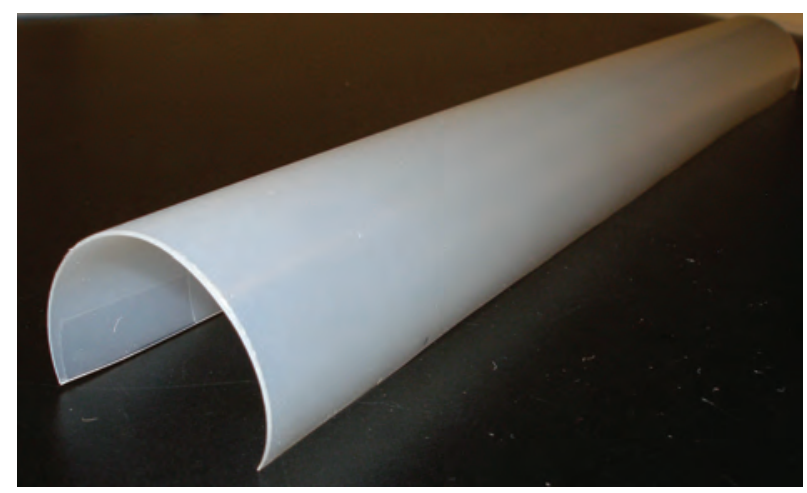

Figure 19: Tape spring made of LDPE.

its straight configuration. The cross section then continued to recover at a slower rate. The whole process involved bending deformation only. Equal sense folding produced a vastly different behavior: the recovery process started with uncoiling followed by a twisting motion. Equal sense folding also took longer to recover the straight configuration, the process took approximately 3 times longer than for opposite sense folding.

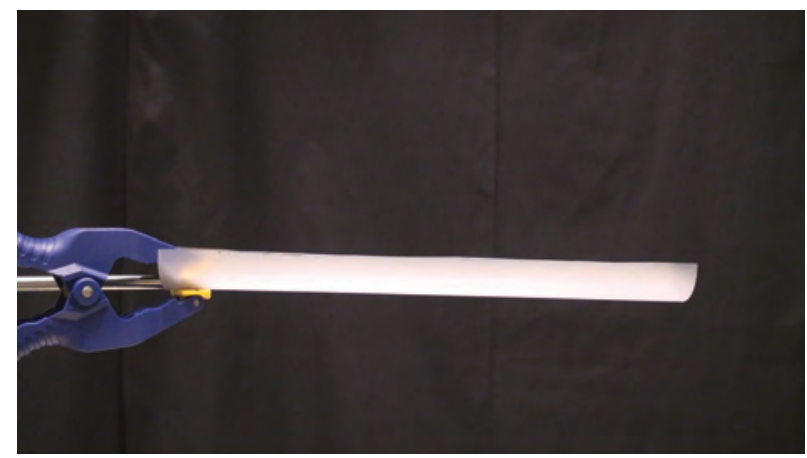

Figure 20: Initial configuration of tape spring.

\section{Conclusions and Future Work}

We have demonstrated the application of a linear isotropic viscoelastic material model in the analysis of the shape recovery of a beam made of LDPE under a combination of prescribed deformation and loading history. The viscoelastic model predicted the change in reaction force and shape over time with high accuracy. We have also investigated experimentally the shape recovery behavior of a LDPE tape spring that was released after being held folded for a period of time.

We are currently extending our viscoelastic material model to capture the behavior of a tape spring through a finite element simulation which will allow us to make quantitative comparisons with the experiment presented in Section IV.B.

\section{Acknowledgment}

We thank Northrop Grumman for financial support. The authors are grateful to Dr Theresa Kidd, Dr Wolfgang Knauss, and Dr Mehran Mobrem for helpful comments and discussions. 


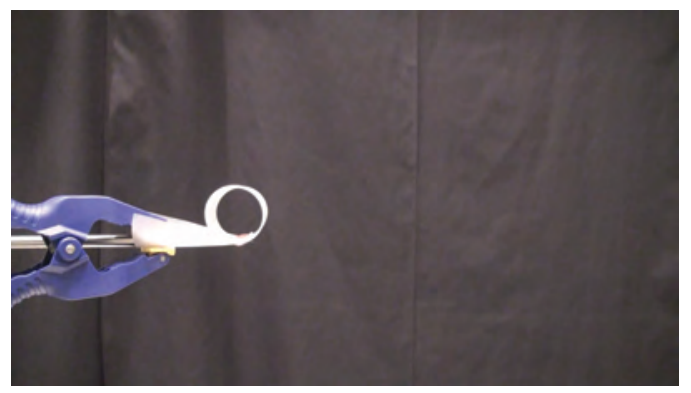

(a)

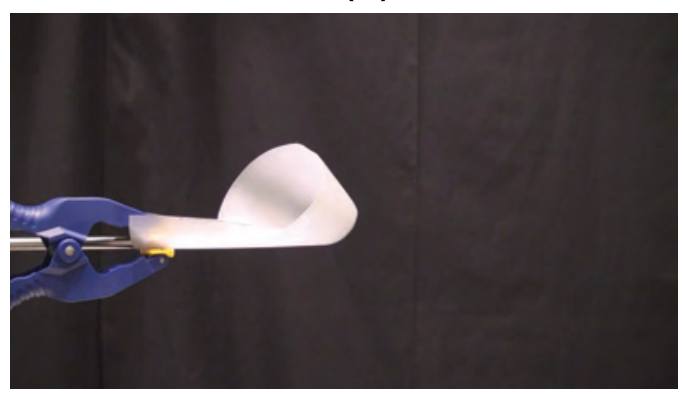

(c)

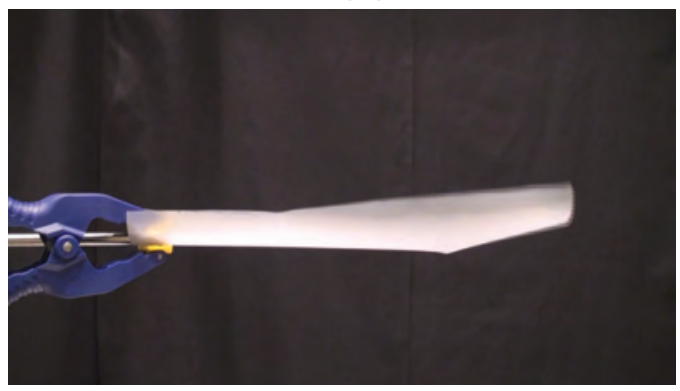

(e)

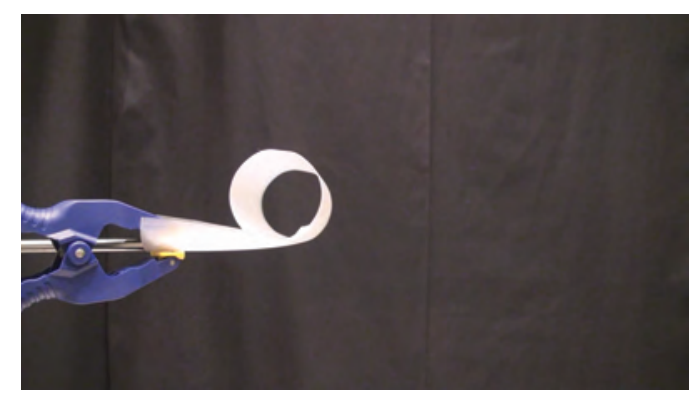

(b)

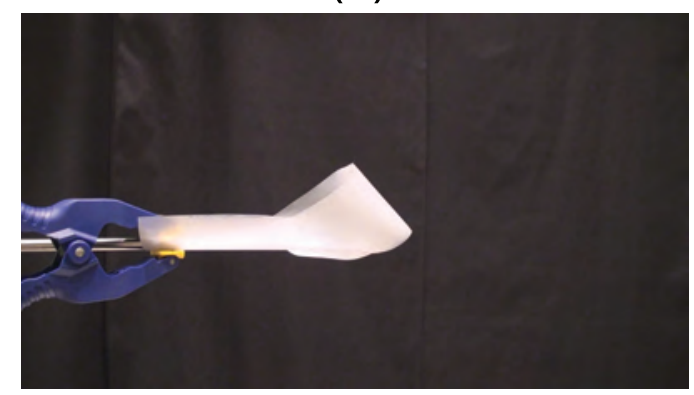

(d)

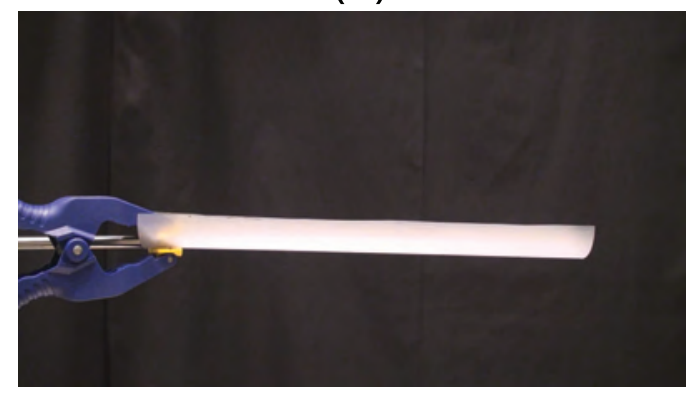

(f)

Figure 21: Equal sense folding: (a) folded; (b) $60 \mathrm{~s}$, (c) $90 \mathrm{~s}$, (d) $120 \mathrm{~s}$, (e) $300 \mathrm{~s}$ and (f) $600 \mathrm{~s}$ after release. 


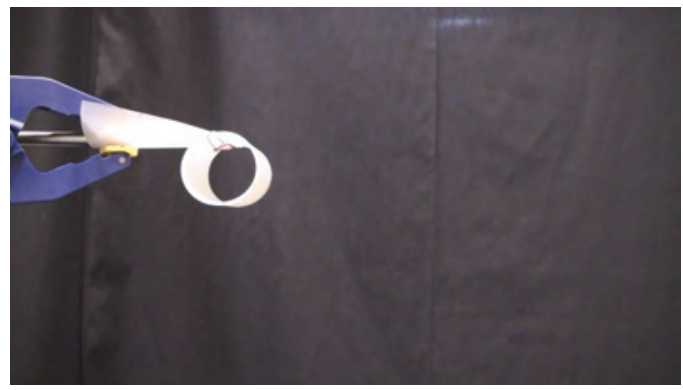

(a)

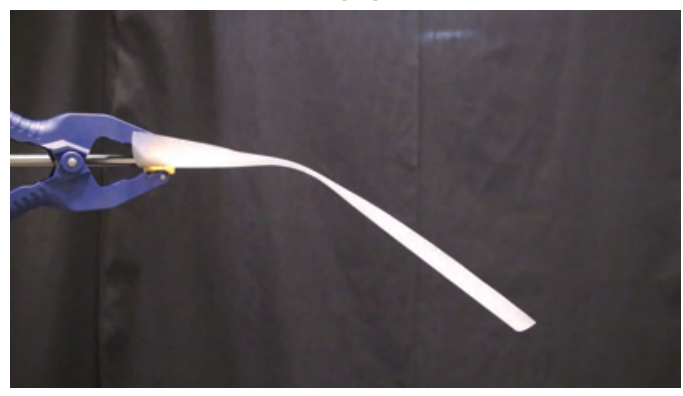

(c)

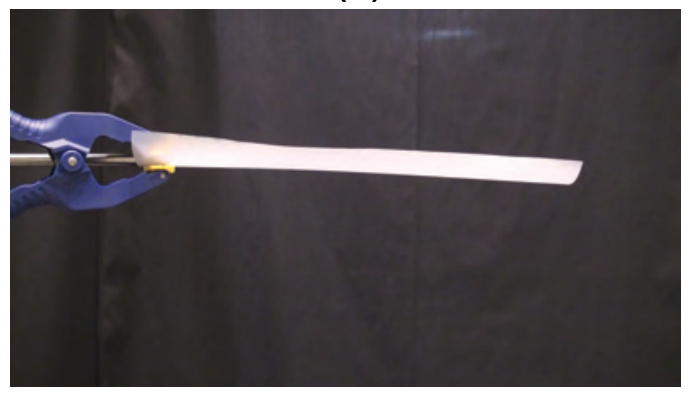

(e)

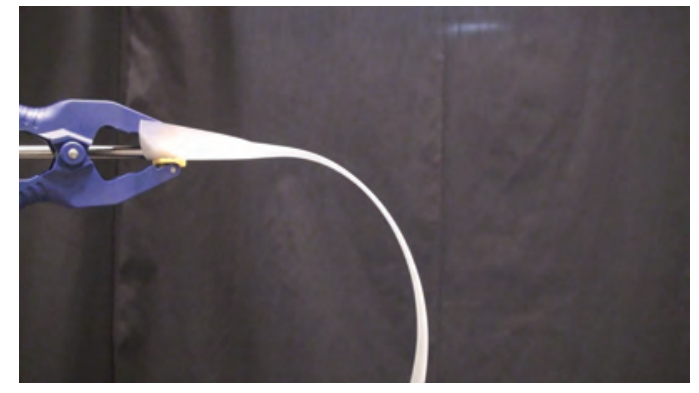

(b)

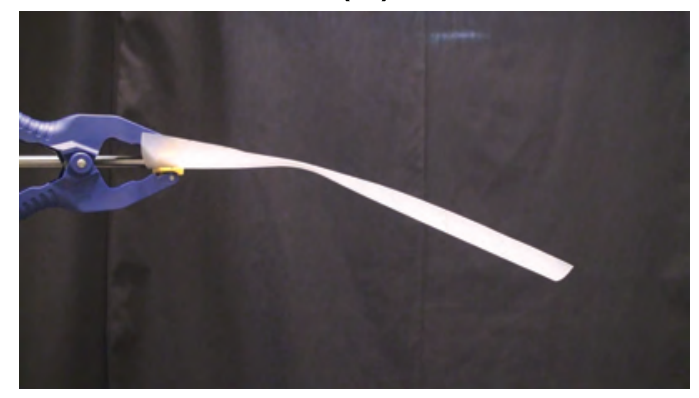

(d)

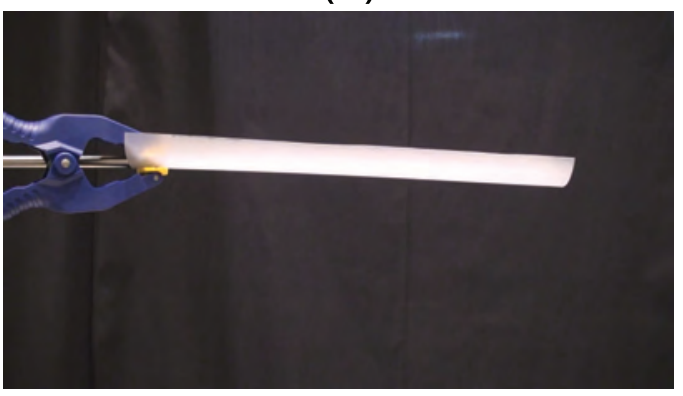

(f)

Figure 22: Opposite sense folding: (a) folded; (b) $30 \mathrm{~s}$, (c) $60 \mathrm{~s}$, (d) $90 \mathrm{~s}$, (e) $120 \mathrm{~s}$ and (f) $600 \mathrm{~s}$ after release. 


\section{References}

${ }^{1}$ Mobrem, M., and Adams, D.S., "Analysis of the lenticular jointed MARSIS antenna deployment," 47th AIAA/ASME/ASCE/AHS/ASC Structures, Structural Dynamics, and Materials Conference, AIAA-2006-1683, Newport, Rhode Island, 1-4 May 2006.

${ }^{2}$ Mobrem, M., and Adams, D.S., "Deployment analysis of lenticular jointed antennas onboard the Mars Express Spacecraft," Journal od Spacecraft and Rockets, Vol. 46, No.2, 2009, pp. 394-402.

${ }^{3}$ Yee, J. C. H., and Pellegrino, S., 'Composite tube hinges,' Journal of Aerospace Engineering, Vol. 18, No.4, 2005, pp. $224-231$.

${ }^{4}$ Aboudi, J., "Mechanics of Composite Materials: A Unified Micromechanical Approach", Elsevier, Amsterdam, 1991.

${ }^{5}$ Muliana, A.H., and Kim, A.S., "A concurrent micromechanical model for nonlinear viscoelastic behaviors of composites reinforced with solid spherical particles," International Journal of Solids and Structures, Vol. 44, 2007, pp. $6891-6913$.

${ }^{6}$ Violette, M.G., Schapery, R.A., "Time-dependent compressive strength of unidirectional viscoelastic composite materials," Mechanics of Time-Dependent Materials, Vol. 6, 2002, pp. 133-145. 2008.

${ }^{7}$ Brinson H.F, and Brinson L.C., Polymer Engineering Science and Viscoelasticity: An Introduction, Springer, New York,

${ }^{8}$ Ferry, J.D., Viscoelastic Properties of Polymers, 3rd ed. John Wiley and Sons, New York, 1980.

${ }^{9}$ Flugge, W., Viscoelasticity. Springer-Verlag, New York, 1974.

${ }^{10}$ SIMULIA, ABAQUS/STANDARD Version 6.7, Providence, RI 